\title{
МОСКОВСКИЙ РЕГИСТР БОЛЬНЫХ АКРОМЕГАЛИЕЙ И ГИПОФИЗАРНЫМ ГИГАНТИЗМОМ. РЕАЛИИ И ПЕРСПЕКТИВЫ
}

\author{
Анциферов М.Б., Алексеева Т.М., Пронин Е.В.
}

ГБУз «Эндокринологический диспансер ДЗМ», Москва

Акромегалия является тяжелым полисистемным заболеванием, успех в лечении которого зависит от своевременной диагностики, дифференцированной лечебной тактики и пожизненного динамического наблюдения. Организационной составляющей специализированной медицинской помощи являются региональные и национальные регистры, позволяющие оптимизировать лабораторно-инструментальную диагностику, обеспечить предиктивное и персонализированное лечение и контролировать адекватность лечебных мероприятий.

ЦЕЛЬ: гарантированное обеспечение каждому пациенту современного уровня диагностического обследования, квалифицированного и преемственного лечения, а также динамического наблюдения.

МАТЕРИАЛЫ И МЕТОДЫ: в настоящее время в Московском регистре числятся 656 пациентов с акро-

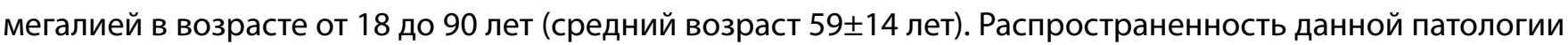
в г. Москве составляет 54 случая/млн, заболеваемость - 4,9 случая.

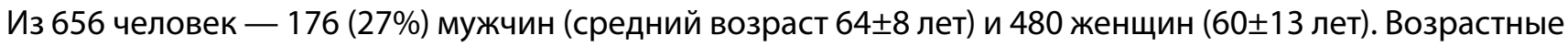
особенности проявляются более агрессивным течением заболевания и резистентностью к проводимой терапии у пациентов с ранним дебютом заболевания по сравнению с лицами старшей возрастной группы. Гендерные различия отмечаются у мужчин с большим объемом аденомы гипофиза (4,9 vs 2,2 cm³), меньшим возрастом диагноза (45,4 vs 49,7 года), более высокими уровнями гормона роста (ГР) (32,6 vs 21,3 нг/мл) и инсулиноподобного ростового фактора (ИРФ-1) (696,5 vs 630,7 нг/мл), p<0,001. Макроаденома гипофиза выявлена у 413 (63\%) больных. В 57 случаях обнаружена смешанная аденома, секретирующая ГР и пролактин (56 чел.), ГР и тиреотропный гормон (1 чел.). Синдром множественных эндокринных неоплазий 1 выявлен у 38 пациентов. Среди наиболее встречающихся осложнений: узловой (смешанный) 306 (54,5\%), артериальная гипертензия (33,5\%), симптоматический сахарный диабет (33\%), онкологические заболевания (19,2\%), полипоз кишечника (10,8\%).

Хирургическое лечение проведено 410 пациентам (62,5\%), первичное - 350, вторичное - 60. 27 больных были оперированы повторно. Средний возраст оперированных пациентов - 44,8 12,8 года. Медикаментозная терапия (МТ) была оказана 489 больным (74,5\%). Первичная МТ — 221, вторичная 268 пациентам. Использовались аналоги соматостатина 1-й генерации [октреотид (191), ланреотид (252)], а также селективный агонист дофамина [каберголин (219)], который в 46 случаях применялся в качестве


Лучевая терапия была проведена 91 пациенту (18 - первичная, 73 - вторичная).

РЕЗУЛЬТАТЫ: радикальная аденомэктомия и полная ремиссия заболевания отмечались в 126 случаях (31\%). У 253 человек проводились вторичная медикаментозная и лучевая терапия. В итоге у 238 больных (58\%) в результате хирургического и комбинированного пособий достигнут клинико-лабораторный контроль акромегалии. Медикаментозный контроль достигнут у 242 больных $(49, \%)$ [при первичной МТ у 109 чел., вторичной - у 133 чел.].

В результате проведенных лечебных мероприятий отмечается положительная биохимическая ди-


$(p=0,001)$. Небольшое превышение показателя обусловлено сохраняющейся проблемой лечения агрессивных аденом. При этом у 69\% больных достигнута стадия контроля акромегалии.

ВЫВоды: дальнейшее совершенствование регистра будет направлено на разработку методов предиктивного и персонализированного лечения с учетом клинико-патологических особенностей ГР-секретирующих аденом и оценку эффективности новых лекарственных препаратов в клинической практике. 